\title{
Genetic polymorphism of miRNA-196a and its target gene annexin- $A 1$ expression based on ethnicity in Pakistani female breast cancer patients
}

\author{
Amena Rahim ${ }^{1}$, Muhammad Afzal ${ }^{2}$, \\ Abdul Khaliq Naveed ${ }^{3}$
}

\begin{abstract}
Objective: To evaluate the association of miR-196a rs11614913 C/T genetic variation and its target gene annexin A1 mRNA expression with breast cancer risk in Pakistani female ethnicities.

Methods: This case control study, conducted from March 2017 to November 2018 included 295 breast cancer patients, 295 controls of three Pakistani ethnicities and archived 100 samples of cohort group for genotyping and expression profiling. Genotyping of miR-196a (rs11614913 C/T) was done by ARMS PCR technique. Annexin-A1 (ANXA1) mRNA expression was measured with qRT-PCR and detection of protein expression of ANXA1 was done by immunohistochemistry.

Results: CC homozygous genotype of miR-196a rs 11614913 was present in $81.4 \%$ of cases and $73.9 \%$ controls. C/T polymorphism was found to be significantly associated with decrease risk of breast cancer $(O R=0.25(0.11-0.58, p<0.05)$. Similar trend was seen with the minor T allele $(O R=0.55(0.39-0.77, p$ $<0.05$, and both dominant and recessive models $(\mathrm{OR}=0.64 ; p=0.02$ and $\mathrm{OR}=0.26, p=0.00)$. In the KPK ethnic group significant decrease association with breast cancer risk was observed $(\mathrm{OR}=0.22(0.09-0.53, p<0.05)$. Immunohistochemical staining showed loss of ANXA1 protein expression in 72 samples, and significant association was observed with pathological type $p=0.00$ and triple negative receptor status $p=0.03$ and with genotypes of miR-196a $p=0.00$. Increase relative expression of $2.81 \pm .88$ by qPCR analysis of ANXA1 mRNA was noted with TT genotype.

Conclusions: Our results demonstrate that miR-196a rs11614913 C/T polymorphism is associated with a decreased risk and loss of protein expression in breast cancer in the Pakistani population.
\end{abstract}

KEYWORDS: Single nucleotide polymorphism; Breast cancer; miR-196a, annexin-A1. ANXA1 gene expression.

How to cite this:

doi: https://doi.org/10.12669/pjms.35.6.1322

Rahim A, Afzal M, Naveed AK. Genetic polymorphism of miRNA-196a and its target gene annexin-A1 expression based on ethnicity in Pakistani female breast cancer patients. Pak J Med Sci. 2019;35(6):1598-1604. doi: https://doi.org/10.12669/pjms.35.6.1322

This is an Open Access article distributed under the terms of the Creative Commons Attribution License (http://creativecommons.org/licenses/by/3.0), which permits unrestricted use, distribution, and reproduction in any medium, provided the original work is properly cited.

Correspondence:

Prof. Amena Rahim, MBBS, FCPS.

Faculty of Basic Medical Sciences,

Biochemistry Department,

Islamic International Medical College,

Riphah International University,

Islamabad-Pakistan.

Email: amena.rahim@riphah.edu.pk

* Received for Publication

* $1^{\text {st }}$ Revision Received:

* $2^{\text {nd }}$ Revision Received:

* Final Revision Accepted:
July 7, 2019

July 16,2019

August 27, 2019

September 7, 2019

\section{INTRODUCTION}

Breast carcinoma is the most common cancer among women. There are 611,000 breast cancer (BC) deaths each year and it was the $18^{\text {th }}$ leading cause of disability-adjusted life-years in 2017. ${ }^{1} \mathrm{~Pa}$ kistan is a developing country with low health care investments. In HAQ index, Pakistan is ranked $154^{\text {th }}$ out of 194 countries in the world. ${ }^{2}$ MicroRNAs are non-coding 20-22 nucleotide long single stranded RNA molecules. They base pair to the 3 untranslated region (3 UTR) of target mRNA to 
regulate gene expression. Most miRNAs are down regulated in cancer cells and this repression causes cellular transformation which can lead to tumor development and progressionmiR-196a (rs11614913) $\mathrm{C}$ to T nucleotide substitution in the 3 'p end of the mature strand sequence can modify its expression and function and this can lead to increased cancer susceptibility. ${ }^{3}$ miR-196a target genes are involved in cell cycle, differentiation and apoptosis. Luthra et al., reported annexin-A1 (ANXA1) as one of the target genes of miR-196a. ${ }^{4}$ ANXA1 regulates physiological mechanisms such as hormone secretion, apoptosis, exocytosis and signal transduction. $m i R$ $196 a$ acts by targeting ANXA1 and suppressing it, thereby promoting cell proliferation and suppressing apoptosis and this provides evidence for its oncogenic potential. ${ }^{5}$ Expression levels of ANXA1 detected in different cancers are not consistent. In certain tumors such as head and neck, esophageal squamous cell and prostate cancers they are down regulated and in others like glioma and oropharngeal cancers they are up regulated. ${ }^{6}$

Pakistan is a multiethnic state with five provinces and different ethnicities. This study was aimed to assess the risk association of miR-196a rs11614913 $\mathrm{C} / \mathrm{T}$ variant and the relationship of $m i R-196 a$ genotype with expression of its target gene ANXA1 in $B C$ cases. Once we get an insight into the pattern of these particular miRNAs, polymorphism in our population it will help the oncologist in detection of breast cancer in its very early stage as individuals with high risk genetic variants of these miRNAs can be screened periodically to avoid un detection or late detection of the breast cancer and they will be able to routinely check for any symptoms without undergoing any invasive tests. According to our information it is the first study to study this association in our population.

\section{METHODS}

A case control study with a non-probability convenient sampling technique was conducted from March 2017 to November 2018. A total of 295 patients from two hospitals - Holy family hospital, Rawalpindi and NORI hospital, Islamabad were included. The Ethical Review Committee of Islamic international Medical College approved the study (Appl.\#Riphah/IRC/18/0356), and informed written consent was taken from the study subjects. Patients were recruited for the study without any restriction on age or disease histology and included three major ethnic groups of Pakistani females including Punjabi, Khyber Pakhtunkhwa and
Kashmiri females. Their demographic and clinical data was collected from the hospital files. They included diagnosed cases, both with family history or sporadic cases of breast cancer; all age groups with different stages of breast tumors. Breast cancer patients and healthy controls were not related but belonged to same ethnic groups. Controls were recruited from within the general population and were frequency matched to cancer patient for age, gender and ethnicity. Their inclusion criteria was; absence of prior history of cancer or any precancerous condition, absence of any chronic disease and females with any first degree relative with breast cancer. Those having any other accompanying malignancy both presently and in the past were excluded. Annexin A1 gene expression was studied from 100 FFPE (Formalin Fixed, paraffin embedded) tissue blocks of clinically diagnosed BC cases with different stages and ages.

Genomic DNA from blood was extracted using $5-7 \%$ chelex (Bio-Rad). The DNA concentration was measured at 260/280 nm (Nano drop 2000c, Thermo Fischer Scientific). The genotyping of $m i R-196 a$ (rs11614913) C/T gene variation was detected by allele specific T-ARMS-PCR technique. ${ }^{7}$ Four primer set, two outer; forward outer used for the amplification of $\mathrm{C}$ and $\mathrm{T}$ alleles. Common Primer -R5 GGCATAAAGCAGGGTTCTCCAGACTTGT-3` and Common Primer F5 GGTCCCATTTCACCAG ATTTTTCCTGAG3 and two inner allele specific; Inner forward C-allele (5'AGTTTTGAACTCGGCAACAAGAAAGTGC-3') and reverse T-allele 5'- CGACGAAAACCGACTGATGTAACTGAGA -3 were used for the amplification of $C$ and $G$ alleles. PCR reactions were performed in a thermal cycler (Major Sciences, USA), in a total volume of $20 \mu 1$ containing $1.5 \mu \mathrm{l}$ (approximately $100 \mathrm{ng} / \mu \mathrm{l}$ ) of DNA template, $4 \mu \mathrm{l}$ master mix $\left(12.5 \mathrm{mM} \mathrm{MgCl}_{2}\right)(5 \mathrm{X}$ FIREPol ${ }^{(\mathrm{R})}$ Master Mix- Solis BioDyne), $1 \mu \mathrm{l}$ primer mix (each $10 \mathrm{pmol} / \mu \mathrm{l}$ ) and PCR grade water up to 14.5 $\mu 1$. PCR cycling conditions were optimized as 1 cycle for holding temperature at $95^{\circ} \mathrm{C}$ for 5 mins; 30 cycles at $95^{\circ} \mathrm{C}$ for 30 secs, $59^{\circ} \mathrm{C}$ for 25 secs, $72{ }^{\circ} \mathrm{C}$ for 30 secs and final extension at $72{ }^{\circ} \mathrm{C}$ for 7 minutes. Separation of amplified products was done on $1.5 \%$ agarose gel which consisted $15 \mu$ l ethidium bromide solution of $0.5 \mu \mathrm{g} / \mu \mathrm{l}$ per $100 \mathrm{ml}$ agarose solution. The resolved DNA bands were documented in gel documentation system (G-Box, syngene USA). The PCR amplicons sizes of miR-196a (rs11614913) C/T were as follows: C- allele153 bp, T-allele $199 \mathrm{bp}$ and $297 \mathrm{bp}$ for internal control bands. Internal control primers were used for the common amplification of 
DNA sequences in genotyping. About $10 \%$ of samples were re-assayed by using primer sequences from two different companies. For the separation of DNA from FFPE tissue samples, a Nucleo Spin kit (NucleoSpin ${ }^{\circledR}$ DNA FFPE XS (MACHEREYNAGEL) was used. The NucleoSpin kit (NucleoSpin ${ }^{\circledR}$ RNA FFPE XS (MACHEREY- NAGEL)) was used for the separation of RNA from FFPE samples following manufacturer's instructions. For RNA reverse transcription to cDNA, the FIREScript RT cDNA Synthesis KIT (Solis BioDyne) cat No; 0824-00001 was used. cDNA synthesized was used for the measurement of annexin-A1 gene expression and after quantification by SYBR green assay (MiniOpticon real-time PCR detection system with CFX Manager ${ }^{\mathrm{TM}}$ software- Bio Rad). mRNA expression levels were normalized to that of $\beta$-actin.

Mouse/Rabbit Immuno-Detector DAB HRP Brown Detection System (Bio SB, USA) was utilized for immunodetection. Antibodies used were Annexin-A1 (MRQ 3) and Mouse Monoclonal Antibody (CELL MARQUE- SIGMA- ALDRICH.) (cat No; 221M-17). Immunohistochemical staining for annexin A1 was carried out on 3-4 $\mu \mathrm{m}$ FFPE embedded breast tissues according to standard protocol. A histopathologist analyzed ANXA1 tissue section staining intensity.

Statistical Analysis: Descriptive statistics for frequency distributions, $\chi^{2}$ test for the association of genotypes with clinicopathological features, allele frequency difference between cases and controls and ANXA1 protein expression. Odds ratio $(\mathrm{OR})$ and $95 \%$ confidence interval $(\mathrm{CI})$ were calculated for the association of genotype, breast cancer risk and ethnicity in cases and controls. Univariate and multivariate logistic regression models were applied. p-value $<0.05$ was taken as significant. Data analysis was done by SPSS version 22.0 (SPSS, Inc; IL, USA).

\section{RESULTS}

Descriptive statistics: The study group consisted of 295 breast cancer cases (median age 56.2 \pm 10.06 years) and 295 controls (median age $56.0 \pm 10.2$ years), sporadic cases (88.1\%) and familial cases $(11.9 \%)$. The left side involvement was 59\%, tumor size $2-5 \mathrm{~cm}(73.9 \%)$, histological type infiltrating ductal carcinoma $(79.3 \%)$, and grade II and stage II ( $82 \%$ and $47.1 \%$ ) respectively.

The genotype frequency of miR-196 a compared with different clinicopathological features: Age, size, pathological type, grade and stage of the disease showed a significant association $(p=0.00)$. Family history, side and receptor status did not show any association.

Genotypic frequency between cases and controls: In dominant model, the minor allele $\mathrm{T}$ showed a decreased association with breast cancer risk $(\mathrm{OR}=0.64,95 \% \mathrm{CI}=0.43-0.95, p=0.02)$ and a similar association existed in the recessive model $(\mathrm{OR}=0.26$, $95 \% \mathrm{CI}=0.11-0.59, p=0.00)$ and in the allelic model (OR=0.55, 95\% CI=0.39-0.77, $p=0.00$; Table-I).

Table-I: Association of miR-196a genotype and allelic frequencies in cases and Controls.

\begin{tabular}{|c|c|c|c|c|}
\hline SNP $196 C>T$ & Cases $N=295$ & Controls $N=295$ & OR $(95 \%$ CI) & $p$-Value \\
\hline Genotype & $n(\%)$ & $n(\%)$ & & \\
\hline $\mathrm{CC}$ & $240(81.4)$ & 218 (73.9) & Ref 1 & \\
\hline CT & 47 (15.9) & 49 (16.6) & $0.87(0.56-1.35)$ & 0.53 \\
\hline \multirow[t]{2}{*}{$\mathrm{TT}$} & $8(2.7)$ & $28(9.5)$ & $0.25(0.11-0.58)$ & 0.00 \\
\hline & \multicolumn{3}{|c|}{ Dominant Model } & \\
\hline $\mathrm{CC}$ & $240(81.4)$ & 218 (73.9) & -- & \\
\hline \multirow[t]{2}{*}{$\mathrm{TT}+\mathrm{CT}$} & 55 (18.6) & $77(26.1)$ & $0.64(0.43-0.95)$ & 0.02 \\
\hline & \multicolumn{3}{|c|}{ Recessive Model } & \\
\hline $\mathrm{CC}+\mathrm{CT}$ & 287 (97.2) & $267(90.5)$ & -- & \\
\hline \multirow[t]{2}{*}{ TT } & $8(2.7)$ & $28(9.5)$ & $0.26(0.11-0.59)$ & 0.00 \\
\hline & & Alleles & & \\
\hline C & $527(89.3)$ & $485(82.2)$ & -- & \\
\hline $\mathrm{T}$ & 63 (10.6) & 105 (17.7) & $0.55(0.39-0.77)$ & 0.00 \\
\hline
\end{tabular}

OR - Odds Ratio, CI- confidence Interval, P $<0.05$ - statistically significant. 
Table-II: Association of miR-196a genotype with ethnicity in cases and control groups.

\begin{tabular}{|c|c|c|c|c|}
\hline Ethnicity/Genotype & Cases $N=295 n(\%)$ & Controls $N=295 n(\%)$ & OR $(95 \% C I)$ & $p$-Value \\
\hline Punjabi & $\mathrm{n}=195(\%)$ & $\mathrm{n}=198(\%)$ & $\mathrm{CC}$ vs TT+CT & \\
\hline $\mathrm{CC}$ & $156(80)$ & $159(80.3)$ & \multirow{2}{*}{$1.01(0.62-1.67)$} & \multirow{2}{*}{0.92} \\
\hline $\mathrm{CT}+\mathrm{TT}$ & $39(20)$ & 39 (19.6) & & \\
\hline Kashmiri & $\mathrm{n}=41(\%)$ & $\mathrm{n}=39(\%)$ & \multirow{4}{*}{$0.46(0.16-1.33)$} & \multirow{4}{*}{0.15} \\
\hline $\mathrm{CC}$ & $34(82.9)$ & $27(69.2)$ & & \\
\hline $\mathrm{CT}+\mathrm{TT}$ & 7 (17) & $12(30.7)$ & & \\
\hline $\mathrm{KPK}^{*}$ & $\mathrm{n}=59(\%)$ & $\mathrm{n}=58(\%)$ & & \\
\hline CC & $50(84.7)$ & $32(55.2)$ & \multirow{2}{*}{$0.22(0.09-0.53)$} & \multirow{2}{*}{0.00} \\
\hline $\mathrm{CT}+\mathrm{TT}$ & $9(15.2)$ & $26(44.8)$ & & \\
\hline
\end{tabular}

A significant association between miR-196a genotype and breast cancer risk was observed only in the Khyber Pukhtunkhwa (KPK) ethnic group $(\mathrm{OR}=0.22,95 \% \mathrm{CI}=0.09-0.53, p=0.00)$. No association was observed in the other two ethnic groups i.e. Punjabi and Kashmiri, Table-II.

\section{Univariate and multivariate analysis:} The patients with genotypes CC (OR=4.39, $95 \% \mathrm{CI}=1.97-9.79, p<0.001)$ and $\mathrm{CT} \quad(\mathrm{OR}=3.55$, $95 \% \mathrm{CI}=1.48-8.52, p=0.004$ ) were found to be more likely to develop breast cancer compared to the TT genotype.

At a univariate level the estrogen receptor (ER), progesterone receptor (PR) and HER2 receptor status were found to be statistically significantly associated with all three genotypes CC, CT and TT $(p<0.001)$, while the combined parameters of ER+PR+HER2 showed no association at the univariate level $(p=0.57)$.

Multinomial logistic model for association with miR-196a genotype, PR was found to have a protective response and was less likely to be associated with the CT genotype (OR=0.21, $95 \% \mathrm{CI}=0.05-0.89, p=0.03$ ).

Out of 100 FFPE tissue samples loss of expression was seen in $72 \%$ cases and positive expression was seen only in $28 \%$ cases. ANXA1 expression showed a highly significant association with pathological type $(p=0.00)$, and triple negative receptor status $(p=0.03)$. However no significant association was seen with age, stage, size and grade.

About $28 \%$ were positive for ANXA1 expression and $72 \%$ showed negative expression $(p=0.00)$. Negative expression of the genotypes CC, CT and TT was $62.5 \%, 34.7 \%$ and $2.8 \%$, respectively. After normalization with reference to the $\beta$-actin gene, mean levels of $33.68 \pm 1.68$ for $\beta$-actin gene and $26.40 \pm 3.88$ for ANXA1 were seen. Fig.1. Immunohistochemical stained breast cancer samples for ANXA1 are shown in Fig.2.

\section{DISCUSSION}

Worldwide incidence of cancer is increasing yearly and the responsible factors are mostly unknown. Genetic factors have been implicated as one of the major cause of cancer, and over the past few decades' microRNAs have established their role in the manifestation of various cancers including breast cancer. MiRNAs are involved in the normal physiological mechanisms, which

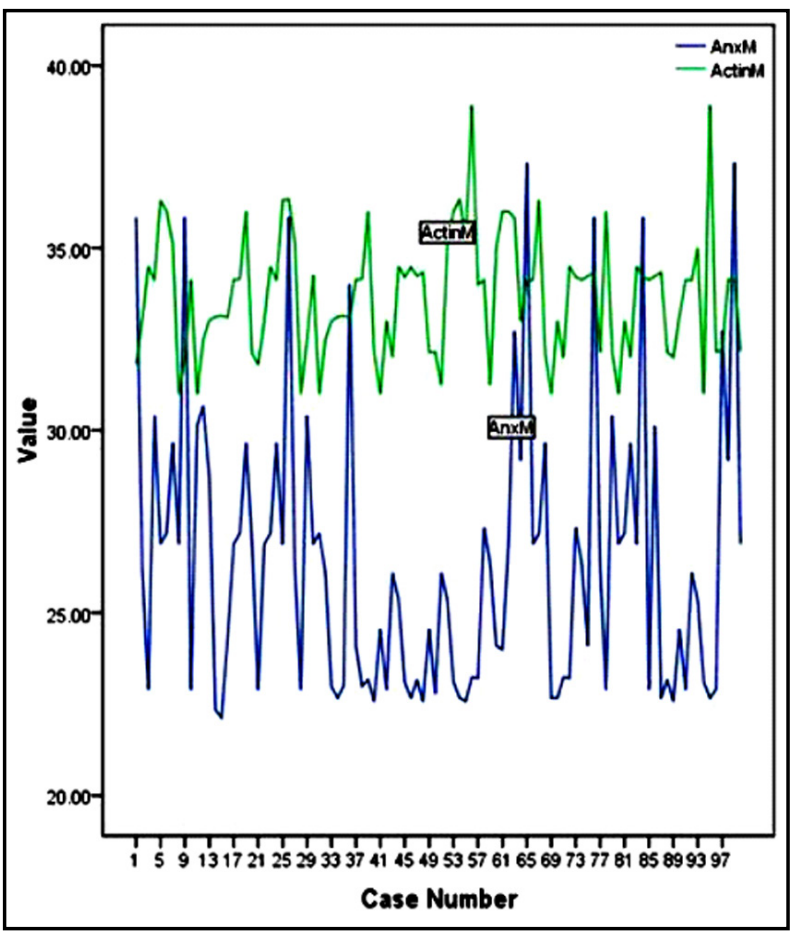

Fig.1: Real time PCR analysis of annexinA1 expression in breast cancer samples in different genotypes of miR-196a. 


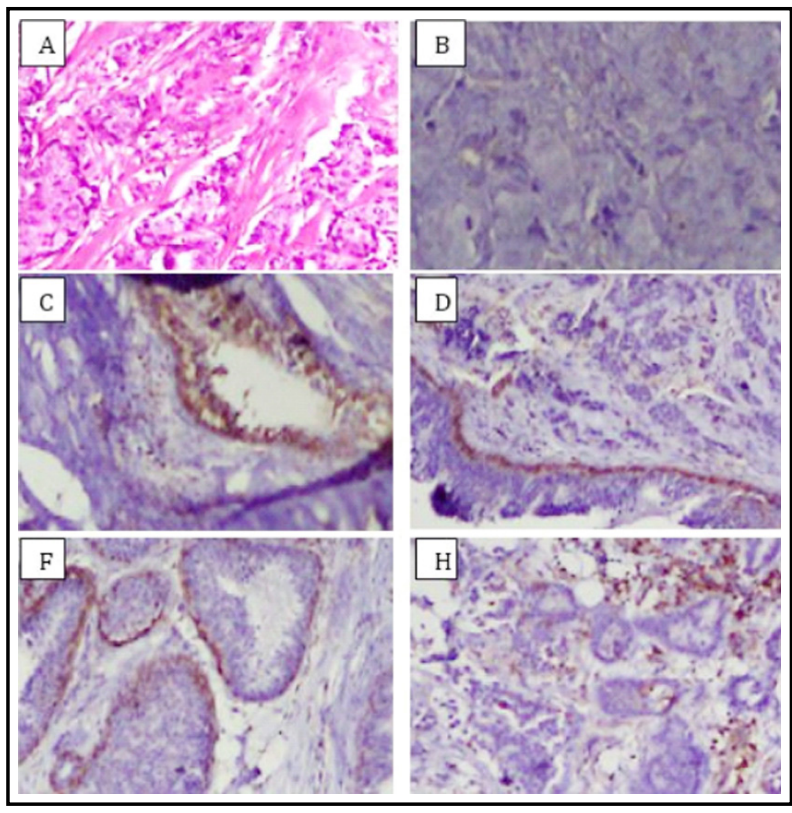

Fig.2: Representive pictures of Annexin A1 (ANXA1) expression in breast tissues. (A) Hematoxylin and eosin stained primary breast cancer tissue. Immunohistochemical stained breast cancer samples for ANXA1 marker (B and C) negative and positive sample built in control. (D) Positive in myoepithelium cells and slight positive in tumor part. ( $\mathrm{F}$ and $\mathrm{H}$ ) ANXA1 expression positive in ductal carcinoma In-situ and primary breast cancer case. 200x magnification.

are regulated by targeting their concerned genes involved in the regulation of various processes like cellular proliferation and apoptosis. Deregulation like single nucleotide polymorphisms may predispose them towards malignant alterations. ${ }^{8}$ miR-196a have been implicated by several studies in different cancers, In vertebrates the gene family for miR-196a is located on chromosome 12 in the region of homeobox clusters which encode homeo domain containing transcription factors. Hox proteins act as anti-apoptotic in BC cells by interfering with p53 genes. miR-196a rs11614913 variant may intensify or diminish the translation of its protein. ${ }^{9}$ According to Alshatwi et al the expression levels of miR-196a (rs11614913) are very high in both pancreatic and breast cancers as compared to normal tissues and this increase expression is associated with decrease survival rates in carcinoma pancreas, and increase chance of developing breast cancer. ${ }^{10} \mathrm{miR}-196 a$ variant not only affects the levels of mature miR-196a but also influences the expression levels of its target gene. ${ }^{11}$ Different studies are conducted to study a link between miR-196a (rs11614913) and breast cancer risk. Some studies report it as a protective factor while others like Omrani et al and Qi P et al observed increase susceptibility to breast cancer. ${ }^{12,13}$ This current study was conducted to study the risk association between miR-196a C/T rs11614913 polymorphism and breast cancer in Pakistani population and its different ethnicities. To date studies done so far have tried to find out the association between this SNP in various populations like Chinese, Caucasians, Italians and Germans etc. ${ }^{14-16}$ In the subgroup analysis the homozygous CC genotype had the highest frequency in all the ethnicities of the Pakistani population in both cases and controls. The frequency of the $\mathrm{C}$ allele was also found to be greater in both the cases and the controls as compared to the $\mathrm{T}$ allele. $\mathrm{C}$ was the major allele in both Brazilian and Iranian populations. ${ }^{17,18}$ Our study confirmed decreased susceptibility to breast cancer with the TT genotype. Hu et al indicated that the TT genotype was protective compared to the $\mathrm{CC} / \mathrm{TT}$ genotype. ${ }^{19}$ When the risk association was analyzed with disease free survival, the C-allele of $m i R-196 a$ exhibited worse prognosis than the others. ${ }^{3}$ Similarly a meta-analysis of 15 studies also correlated CC genotype with increased risk of breast cancer in comparison to the TT genotype. ${ }^{20}$ When analyzed by ethnicity, it was observed that there was a significant association with BC only in the population with KPK ethnicity. It means that SNPs show diversity among and within the ethnic groups of a population. Meta-analysis observed decreased risk of $\mathrm{BC}$ in both allelic and recessive models, ${ }^{21}$ and another analysis indicated an increased association with cancer susceptibility of the homozygous CC genotype among Asians and a decreased risk among Caucasians. ${ }^{14}$ No such relationship was observed in Australian, ${ }^{15}$ German and Italian women. ${ }^{16}$ In contrast $\mathrm{Hu}$ et al. ${ }^{19}$ established an increased association and Hoffman et al. ${ }^{11}$ evidenced a decreased association. The contradiction in our study can be justified by factors related to the population, which includes the genetic backgrounds of different ethnic groups. Our genetic makeup does not belong to Chinese races in Asia. Our two ethnic groups in this study are more related to the Indian genetic model, whereas those from KPK are related to Jewish and Greek ancestry. ${ }^{22}$

ANXAA1 is calcium dependent phospholipid binding proteins that are involved in cancer and metastasis. In different cancers ANXA1 expressions are either up regulated or down regulated. $m i R-196 a$ can act as a tumor suppressor, 
as well as an oncogene by directly targeting the HOX genes and ANXA1 genes, which in turn by a negative feedback loop affect the miR-196a expression levels in breast cancer. ${ }^{23}$

We tried to establish the relationship of miR$196 a$ polymorphism and mRNA expression of its target gene ANXA1 with clinicopathological features, receptor status and genotype in our population. Changes in the expression levels of ANXA1 were noted in pathological type, and with triple negative receptor status. This means that it's low or absent expression levels are associated with poor prognosis. Wang et al. observed low expressions in $56.3 \%$ of cases and more negative expression in advance disease stage. ${ }^{24}$ Cao et al. noted down regulation of ANXA1 in $79 \%$ of BC cases. $^{5}$ While Deng et al. observed 80\% down regulation. ${ }^{25}$

Limitations of the study: In depth analysis of the mechanism of action of miR-196a and its various genotypes on its target genes warrants further study. The strength of this study is that, for the first time, we have assessed the genotypes of miR-196a in the Pakistani population and its relationship to cancer susceptibility along with its target gene ANXA1.

\section{CONCLUSIONS}

Our study showed that the miR-196a (rs11614913) $\mathrm{C} / \mathrm{T}$ polymorphism produced a decreased susceptibility to breast cancer different ethnicities of Pakistani population. ANXA1 expression is low in breast cancer and negative expression is associated with the homozygous CC genotype and the C/T variant of miR-196a rs11614913.

Acknowledgements: We are grateful to the Director Dr. Mohammad Faheem and staff of NORI, hospital Islamabad, Prof. Dr. Muhammad Ayub ex-commandant AFIP, and Prof. Jamal Nasir pathologist, for their help and guidance in sample collection.

\section{Conflict of interest: None.}

Source of funding: None.

\section{REFERENCES}

1. Roth GA, Abate D, Abate KH, Abay SM, Abbafati C, Abbasi $\mathrm{N}$, et al. Global, regional, and national agesex-specific mortality for 282 causes of death in 195 countries and territories, 1980-2017: a systematic analysis for the Global Burden of Disease Study 2017. Lancet. 2018;392(10159):1736-1788. doi: 10.1016/S01406736(18)32203-7
2. Fullman N, Yearwood J, Abay SM, Abbafati C, AbdAllah F, Abdela J, et al. Measuring performance on the Healthcare Access and Quality Index for 195 countries and territories and selected subnational locations: A systematic analysis from the Global Burden of Disease Study 2016. Lancet. 2018;391(10136):2236-2271. doi: 10.1016/S01406736(18)30994-2

3. Lee SJ, Seo JW, Chae YS, Kim JG, Kang BW, Kim WW, et al. Genetic polymorphism of miR-196a as a prognostic biomarker for early breast cancer. Anticancer Res. 2014:34(6):2943-2949.

4. Luthra R, Singh RR, Luthra MG, Li YX, Hannah C, Romans AM, et al. MicroRNA-196a targets annexin A1: a microRNAmediated mechanism of annexin A1 down regulation in cancers. Oncogene. 2008;27(52):6667-6678. doi: 10.1038/ onc.2008.256

5. Cao Y, Li Y, Edelweiss M, Arun B, Rosen D, Resetkova E, et al. Loss of Annexin A1 Expression in Breast Cancer Progression. Appl Immunohistochem Mol Morphol. 2008;16(6):530-534. doi: 10.1097/ PAI.0b013e31817432c3

6. Cai T, Guan X, Wang H, Fang Y, Long J, Xie X, et al. MicroRNA-26a regulates ANXA1, rather than DAL-1, in the development of lung cancer. Oncol Lett. 2018;15(4):58935902. doi: 10.3892/ol.2018.8048

7. Afzal M, Rahim A, Naveed AK, Ahmed S, Kiyani MM. Development of Cost-effective Tetra-primer Amplification Refractory Mutation System (T-ARMS) PCR for the Detection of miR-146a gene rs2910164 C/G Polymorphism in Breast Cancer. Biochem Mol Biol J. 2018;04(01).

8. Hu Y, Yu C-Y, Wang J-L, Guan J, Chen H-Y, Fang J-Y. MicroRNA sequence polymorphisms and the risk of different types of cancer. Sci Rep. 2014;4:3648. doi: 10.1038 /srep03648

9. Eslami-S Z, Tahmaseb M, Ghaderi A. The investigation of miR-196a2 rs11614913 with breast cancer susceptibility in south of IRAN. Meta Gene. 2018;17:4347. doi: 10.1016/j.mgene.2018.04.007

10. Alshatwi AA, Shafi G, Hasan TN, Syed NA, Al-Hazzani AA, Alsaif MA, et al. Differential Expression Profile and Genetic Variants of MicroRNAs Sequences in Breast Cancer Patients. Zhang B, editor. PLoS One. 2012;7(2):e30049. doi: 10.1371/journal.pone.0030049

11. Hoffman AE, Zheng T, Yi C, Leaderer D, Weidhaas J, Slack F, et al. microRNA miR-196a-2 and Breast Cancer: A Genetic and Epigenetic Association Study and Functional Analysis. Cancer Res. 2009;69(14):5970-5977. doi: 10.1158/0008-5472. CAN-09-0236

12. Omrani M, Hashemi M, Eskandari-Nasab E, Hasani S-S, Mashhadi MA, Arbabi F, et al. hsa-mir-499 rs3746444 gene polymorphism is associated with susceptibility to breast cancer in an Iranian population. Biomark Med. 2014;8(2):259267. doi: $10.2217 /$ bmm. 13.118

13. Qi P, Wang L, Zhou B, Yao W, Xu S, Zhou Y, et al. Associations of miRNA polymorphisms and expression levels with breast cancer risk in the Chinese population. Genet Mol Res. 2015;14(2):6289-6296. doi: 10.4238/2015. June.11.2

14. Zhang $\mathrm{H}$, Zhang $\mathrm{Y}$, Yan W, Wang W, Zhao X, Ma X, et al. Association between three functional microRNA polymorphisms (miR-499 rs3746444, miR-196a rs11614913 and miR-146a rs2910164) and breast cancer risk: a metaanalysis. Oncotarget. 2017;8(1):393-407. doi: 10.18632/ oncotarget.13426 
15. Jedlinski DJ, Gabrovska PN, Weinstein SR, Smith RA, Griffiths LR. Single Nucleotide Polymorphism in hsamir-196a-2 and Breast Cancer Risk: A Case Control Study. Twin Res Hum Genet. 2011;14(05):417-21. doi: 10.1375/ twin.14.5.417

16. Catucci I, Yang R, Verderio P, Pizzamiglio S, Heesen $\mathrm{L}$, Hemminki K, et al. Evaluation of SNPs in miR-146a , miR196a2 and miR-499 as low-penetrance alleles in German and Italian familial breast cancer cases. Hum Mutat. 2010;31(1):E1052-E1057. doi: 10.1002/humu.21141

17. Linhares JJ, Azevedo M, Siufi AA, de Carvalho CV, Wolgien MDCGM, Noronha EC, et al. Evaluation of single nucleotide polymorphisms in microRNAs (hsamiR-196a2 rs11614913 C/T) from Brazilian women with breast cancer. BMC Med Genet. 2012;13:119. doi: 10.1186/1471-2350-13-119

18. Mashayekhi S, Saeidi Saedi H, Salehi Z, Soltanipour S, Mirzajani E. Effects of miR-27a, miR-196a2 and miR-146a polymorphisms on the risk of breast cancer. Br J Biomed Sci. 2018;75(2):76-81. doi: 10.1080/09674845.2017.1399572

19. Hu Z, Liang J, Wang Z, Tian T, Zhou X, Chen J, et al. Common genetic variants in pre-microRNAs were associated with increased risk of breast cancer in Chinese women. Hum Mutat. 2009;30(1):79-84. doi: 10.1002/ humu. 20837

20. Chu H, Wang M, Shi D, Ma L, Zhang Z, Tong N, et al. Hsa-miR-196a2 Rs11614913 Polymorphism Contributes to Cancer Susceptibility: Evidence from 15 Case-Control Studies. Creighton C, editor. PLoS One. 2011;6(3):e18108. doi: 10.1371/journal.pone.0018108

21. $\mathrm{Mu} \mathrm{K}, \mathrm{Wu} Z-Z, \mathrm{Yu} J-\mathrm{P}, \mathrm{Guo} \mathrm{W}, \mathrm{Wu} \mathrm{N}$, Wei L-J, et al. Meta-analysis of the association between three microRNA polymorphisms and breast cancer susceptibility. Oncotarget. 2017;8(40):68809-68824. doi: 10.18632/ oncotarget.18516
22. Qamar R, Ayub Q, Mohyuddin A, Helgason A, Mazhar $\mathrm{K}$, Mansoor A, et al. Y-chromosomal DNA variation in Pakistan. Am J Hum Genet. 2002;70(5):1107-1124. doi: $10.1086 / 339929$

23. Yuan Y, Anbalagan D, Lee LH, Samy RP, Shanmugam MK, Kumar AP, et al. ANXA1 inhibits miRNA-196a in a negative feedback loop through NF-kB and c-Myc to reduce breast cancer proliferation. Oncotarget. 2016;7(19):27007-27020. doi: 10.18632/oncotarget.8875

24. Wang LP, Bi J, Yao C, Xu XD, Li XX, Wang SM, et al. Annexin A1 expression and its prognostic significance in human breast cancer. Neoplasma. 2010;57(3):253-259.

25. Deng S, Wang J, Hou L, Li J, Chen G, Jing B, et al. Annexin A1, A2, A4 and A5 play important roles in breast cancer, pancreatic cancer and laryngeal carcinoma, alone and/or synergistically. Oncol Lett. 2013;5(1):107-112. doi: 10.3892/ ol.2012.959

\section{Authors' Contribution:}

AR did concept, design and manuscript writing, is responsible for integrity of research.

MA conducted experimental work, data analysis. AKN did review and final approval of manuscript.
Authors:

1. Prof. Amena Rahim

2. Dr. Muhammad Afzal Research Officer,

3. Prof. Abdul Khaliq Naveed Department of Biochemistry, CMH Lahore Medical College, National University of Medical Sciences, Islamabad, Pakistan.

1, 2: Department of Biochemistry, Islamic International Medical College, Riphah International University, Islamabad, Pakistan. 